\title{
Porphyromonas gingivalis Induces Apoptosis and Autophagy via ER Stress in Human Umbilical Vein Endothelial Cells
}

\author{
Masaaki Hirasawa and Tomoko Kurita-Ochiai (iD \\ Department of Microbiology and Immunology, Nihon University School of Dentistry at Matsudo, Matsudo-shi, Chiba-ken, Japan \\ Correspondence should be addressed to Tomoko Kurita-Ochiai; ochiai.tomoko@nihon-u.ac.jp
}

Received 5 March 2018; Revised 3 May 2018; Accepted 13 June 2018; Published 29 July 2018

Academic Editor: Sandra Helena Penha Oliveira

Copyright (C) 2018 Masaaki Hirasawa and Tomoko Kurita-Ochiai. This is an open access article distributed under the Creative Commons Attribution License, which permits unrestricted use, distribution, and reproduction in any medium, provided the original work is properly cited.

\begin{abstract}
It has been reported that periodontitis is associated with an increased risk of atherosclerosis. Accumulating evidence suggests that endothelial dysfunction is an early marker for atherosclerosis. To determine how periodontal infections contribute to endothelial dysfunction, we examined the effect of Porphyromonas gingivalis on human umbilical vein endothelial cells (HUVEC). P. gingivalis significantly suppressed the viability of HUVEC, induced DNA fragmentation and annexin V staining, and increased caspase-3, caspase-8, and caspase-9 activities. P. gingivalis also increased the expression of GADD153 and GRP78 and caspase-12 activity. Further, $P$. gingivalis induced autophagy, as evidenced by increased LC3-II and Beclin-1 levels. The suppression of $P$. gingivalisinduced autophagy by silencing of LC3 with siRNA significantly increased $P$. gingivalis-induced apoptosis. ER stress inhibitor, salubrinal, suppressed apoptosis and autophagy by inhibiting P. gingivalis-induced DNA fragmentation and LC3II expression. These data suggest that $P$. gingivalis infection induces ER stress-mediated apoptosis followed by autophagic response that protects HUVEC from $P$. gingivalis-mediated apoptosis, potentially amplifying proatherogenic mechanisms in the perturbed vasculature.
\end{abstract}

\section{Introduction}

Periodontal disease is highly prevalent, affecting up to $90 \%$ of the global population [1]. Porphyromonas gingivalis, a major periodontal pathogen, was recently implicated in the pathogenesis of atherosclerosis [2]. P. gingivalis can directly access the systemic circulation and the endothelium in patients with periodontitis, as transient bacteremias are common [3]. Indeed, $P$. gingivalis has been detected in human atherosclerotic plaques $[4,5]$, and it can both invade endothelial cells (EC) and persist therein [6]. Further, P. gingivalis elicits a proatherogenic response in EC in the form of increased leukocyte adhesion with concomitant upregulation of adhesion molecules and proinflammatory cytokines and chemokines $[7,8]$. Interestingly, these effects require the invasion of EC by viable bacteria [8].

EC are key cellular components of blood vessels that function as a selectively permeable barrier between blood and tissue. It is believed that atherogenic risk factors induce apoptosis in EC, leading to the denudation or dysfunction of the intact endothelial monolayer, with subsequent atherosclerotic lesion formation as a result of lipid accumulation, monocyte adhesion, and inflammatory reactions $[9,10]$. Endothelial cell apoptosis has several potential deleterious effects, including plaque erosion and thrombosis [11]. Recent studies have demonstrated increased endoplasmic reticulum (ER) stress protein expression in the vascular cells of atherosclerotic lesions and regulation of the protein in the endothelium by several atherosclerotic stressors [12].

Autophagy is a cellular defense mechanism involving degradation and recycling of cytoplasmic components. Autophagy can protect cells from apoptosis; thus, it is said to sit at the crossroad between cell death and survival. However, excessive autophagy can destroy essential cellular components and cause cell death [13]. Accumulating evidence has also suggested that ER stress is linked to autophagy [14]. In the present study, we examined the capacity of whole, viable $P$. gingivalis to induce cell death via apoptosis, ER stress, and autophagy in human umbilical vein endothelial cells (HUVEC). 


\section{Materials and Methods}

2.1. Bacterial Strains and Culture Methods. P. gingivalis strain 381 and KDP136 (gingipain-null mutant) were cultured on anaerobic blood agar plates (Becton Dickinson Co., Sunnyvale, CA) in a model 1024 anaerobic system (Forma Scientific, Marietta, $\mathrm{OH}$ ) under $10 \% \mathrm{H}_{2}, 80 \% \mathrm{~N}_{2}$, and $10 \%$ $\mathrm{CO}_{2}$ for 3-5 days. The cells were then inoculated into brain heart infusion broth (Difco Laboratories, Detroit, MI) supplemented with $5 \mu \mathrm{g} / \mathrm{ml}$ hemin and $0.4 \mu \mathrm{g} / \mathrm{ml}$ menadione and cultured to an $\mathrm{OD}_{660}$ of $0.8\left(10^{9} \mathrm{cfu} / \mathrm{ml}\right)$. The cells were then harvested by centrifugation at $8000 \times \mathrm{g}$ for $20 \mathrm{~min}$ at $4^{\circ} \mathrm{C}$ and diluted in phosphate-buffered saline (PBS).

2.2. Cell Line and Reagents. HUVEC (provided by LonzaTakara, Tokyo, Japan) were cultured at $37^{\circ} \mathrm{C}$ in a humidified atmosphere with $5 \% \mathrm{CO}_{2}$ in endothelial cell culture medium (EGM-2 BulletKit, Lonza-Takara). All experiments were performed on cells at passages 4-8 at approximately $80 \%$ confluence.

2.3. Cell Viability Assay and Cytotoxicity Assay. Cell viability was determined using a Cell Counting Kit-8 (CCK-8; Wako, Osaka, Japan). Briefly, cells $\left(1.0 \times 10^{4} /\right.$ well $)$ were cultured in $100 \mu \mathrm{l}$ of endothelial cell culture medium in a 96-well plate and stimulated with $P$. gingivalis at the indicated multiplicity of infection (MOI). After $19 \mathrm{~h}, 10 \mu \mathrm{l}$ of CCK- 8 solution was added, and the cells were incubated at $37^{\circ} \mathrm{C}$ for $2 \mathrm{~h}$, followed by measurement of A450 using a spectrophotometer.

2.4. Measurement of Cell Death. Cellular apoptosis was quantified by DNA fragmentation using the Cell Death Detection ELISA $^{\text {PLUS }}$ kit (Roche Diagnostics, Mannheim, Germany). Briefly, HUVEC $\left(5 \times 10^{5} /\right.$ dish $)$ were cultured with $P$. gingivalis 381 and KDP136 strains at the indicated MOI. After 7,16 , and $24 \mathrm{~h}$, the cells were lysed in $200 \mu \mathrm{l}$ of lysis buffer, and $20 \mu \mathrm{l}$ of the supernatant was reacted with $80 \mu \mathrm{l}$ of anti-DNA immunocomplex conjugated with peroxidase, which interacts with streptavidin-coated wells, in a microtiter plate for $2 \mathrm{~h}$. At the end of the incubation, $100 \mu \mathrm{l}$ of substrate was added, and color development was quantified as a wavelength of $405 \mathrm{~nm}$. The results were calculated as the ratio of the absorbance of the $P$. gingivalis-treated cells to the absorbance of the nontreated control cells. For the apoptosis inhibition assays, cells were preincubated for $1 \mathrm{~h}$ with caspase-12 inhibitor Z-VAD-FMK (MBL, Nagoya, Japan) or ER stress inhibitor, salbrinal $(50 \mu \mathrm{M})$ (Sigma-Aldrich, St. Louis, MO, USA), before stimulation with the bacteria. Apoptosis of the $P$. gingivalis-treated HUVEC was assessed by annexin V-EnzoGold and 7-amino-actinomycin D (7AAD) staining (Enzo Life Sciences Inc., Farmingdale, NY) and flow cytometric analysis using a FACSCalibur Flow Cytometer (Becton Dickinson, Franklin Lakes, NJ).

2.5. Caspase Assay. After incubation $\left(5 \times 10^{5}\right.$ cells/dish) for 16 h with $P$. gingivalis 381, the cells were harvested, and the caspase-1, caspase- 3 , caspase- 8 , caspase- 9 , and caspase- 12 activities were measured using a caspase fluorometric protease assay kit (MBL). The amount of 7-amino-4-trifluoromethylcoumarine (AFC) released was measured using an
ARVO multilabel/luminescence counter with excitation and emission at 400 and $505 \mathrm{~nm}$, respectively.

2.6. Real-Time Quantitative RT-PCR. Quantitative RTPCR was performed using primers specific for $\mathrm{C} / \mathrm{EBP}$ homologous protein (CHOP)/growth arrest and DNA damage 153 (GADD153) (GGCAGCTGAGTCATTGCC and GCAGATTCACCATTCGGTCA), glucose-regulated protein-78 (GRP78) (CCTAGCTGTGTCAGAATCTCCAT CC and GTTTCAATGTCACCATCCAAGATCC), Beclin-1 (CCAGATGCGTTATGCCCAGAC and CATTCCATTCC ACGGGAACAC), microtubule-associated protein 1 light chain 3B (MAP1LC3B) (ACGCATTTGCCATCACAGTTG and GGGACCTTCAGCAGTTTACAGTCAG), and $\beta$-actin (CATCCGTAAAGACCTCTATGCCAAC and ATGGAG CCACCGATCCACA). The thermal cycling profile was as follows: $95^{\circ} \mathrm{C}$ for $10 \mathrm{~s}$ followed by 40 cycles of $95^{\circ} \mathrm{C}$ for $5 \mathrm{~s}$ and $60^{\circ} \mathrm{C}$ for $30 \mathrm{~s}$, with a final dissociation at $95^{\circ} \mathrm{C}$ for $5 \mathrm{~s}$, $60^{\circ} \mathrm{C}$ for $30 \mathrm{~s}$, and $95^{\circ} \mathrm{C}$ for $15 \mathrm{~s}$. The starting amount of RNA was quantified using a standard curve; fold changes in the expression of CHOP/GADD153, GRP78, Beclin-1, and $\mathrm{LC} 3 \mathrm{~B}$ relative to $\beta$-actin were determined in triplicate.

2.7. Antibodies and Western Blot Analysis. Rabbit antibodies against GADD153, GRP78, Beclin-1, and $\beta$-actin were purchased from Santa Cruz Biotechnology Inc. (Santa Cruz, CA), while those against LC-3 were purchased from MBL. Secondary horseradish peroxidase- (HRP-) conjugated goat anti-rabbit and goat anti-mouse antibodies were obtained from Amersham Pharmacia Biotech (Piscataway, NJ). Cells were lysed in a buffer containing $10 \mathrm{mM}$ Tris- $\mathrm{HCl}$, $150 \mathrm{mM} \mathrm{NaCl}, 1 \%$ Nonidet P-40, $1 \mathrm{mM}$ ethylenediaminetetraacetic acid (EDTA), $1 \mathrm{mM}$ ethylene glycol tetraacetic acid (EGTA), $0.1 \mathrm{mM}$ phenylmethylsulfonyl fluoride, $8 \mu \mathrm{g} / \mathrm{ml}$ aprotinin, and $2 \mu \mathrm{g} / \mathrm{ml}$ leupeptin ( $\mathrm{pH} 7.4$ ). For immunoblotting, proteins resolved by $12.5 \%$ sodium dodecyl sulfate-polyacrylamide gel (SDS-PAGE) were transferred to polyvinylidene fluoride membranes (Millipore, Bedford, MA), which were then exposed to primary and then secondary antibodies. Chemiluminescence detection was performed with an ECL ${ }^{\mathrm{TM}}$ Western Blotting Detection Kit (Amersham). The signal intensities of the corresponding bands were measured by a Light Capture equipped with CS Analyzer software (ATTO, Osaka, Japan).

2.8. siRNA Knockdown. Knockdown of endogenous LC3 with siRNA was carried out using Lipofectamine RNAiMAX (Invitrogen) according to the manufacturer's instructions. Briefly, HUVEC grown to $50-80 \%$ confluence in a 6 -well plate were transfected with either LC3B siRNA (sc-43391, Santa Cruz Biotechnology) or control siRNA (sc-37007, Santa Cruz Biotechnology). The final concentration of respective siRNA was $50 \mathrm{nM}$ for each transfection, and the experiments were carried out $24 \mathrm{~h}$ after transfection.

2.9. Acridine Orange Staining. Cells were treated with P. gingivalis 381 at an MOI $1: 10^{2}$ for $8 \mathrm{~h}$ and stained with $1 \mathrm{mg} / \mathrm{ml}$ acridine orange at room temperature for 20 min. Then, cells were washed with PBS and visualized by fluorescence microscopy. 
2.10. Statistical Analysis. All data are presented as means \pm SEM. Multiple-group comparisons were made by one-way analysis of variance, followed by post hoc intergroup comparison by the Bonferroni-Dunn test. A $p$ value $<0.05$ was considered statistically significant.

\section{Results}

3.1. P. gingivalis 381 Inhibits Cell Proliferation and Induces Cell Death through Apoptosis. P. gingivalis 381 significantly diminished the proliferation of HUVEC at higher MOI (Figure 1(a)). Next, we investigated whether the suppression of cell proliferation observed in HUVEC treated with $P$. gingivalis 381 was dependent on apoptosis. Incubation with $P$. gingivalis 381 strongly induced apoptosis in the HUVEC in a dose- and time-dependent manner (Figure 1(b)). Higher apoptosis rates were observed at MOI of $1: 5 \times 10^{2}$ after $16 \mathrm{~h}$ and $1: 10^{2}$ and $1: 5 \times 10^{2}$ after $24 \mathrm{~h}$ compared to the nontreated control group. In contrast, KDP 136 strain did not cause DNA fragmentation in any MOI and $24 \mathrm{~h}$ culture. In addition, we confirmed the possible apoptotic effect of $P$. gingivalis 381 by annexin $\mathrm{V}$ and 7-AAD staining and flow cytometric analysis (Figure 1(c)). The early apoptotic cells represented by the lower right quadrant (7-AAD negative and annexin $\mathrm{V}$ positive) and the nonviable necrotic and late-state apoptotic cells represented by the upper right quadrant (positive for annexin $\mathrm{V}$ binding and 7-AAD uptake). After $21 \mathrm{~h}$ of treatment with $P$. gingivalis 381 at an MOI of $1: 10^{2}$, the number of early-stage apoptotic cells was significantly increased by up to $47.8 \pm 2.0 \%(p<0.01)$. These results indicated that the decrease in viable cells induced by $P$. gingivalis 381 treatment was secondary to apoptosis. Apoptotic cell death typically occurs via the stimulation of caspase activity [15]. Caspase- 8 and caspase- 9 appear to be activated in the death receptorand mitochondrial-dependent apoptotic pathways, respectively, and caspase-3, which is common to both pathways, induces DNA fragmentation. Pyroptosis, which is uniquely dependent on caspase-1, has been described in monocytes, macrophages, and dendritic cells infected with a range of microbial pathogens such as Salmonella, Francisella, and Legionella [16]. Therefore, we assessed the activities of caspase-1, caspase-3, caspase-8, and caspase-9. P. gingivalis 381 enhanced caspase-3, caspase-8, and caspase- 9 activities, suggesting involvement of the mitochondriamediated intrinsic pathway and death receptor-induced extrinsic pathway (Figure $1(\mathrm{~d})$ ). On the other hand, caspase- 1 activity was not enhanced by $P$. gingivalis 381 within the range of the MOI investigated (data not shown). Therefore, cell death induced by $P$. gingivalis 381 was unrelated to pyroptosis.

3.2. ER Stress Is Involved in $P$. gingivalis 381-Induced Apoptosis. To determine whether ER-mediated events contribute to $P$. gingivalis 381-induced apoptosis, we examined the levels of several substances reported to participate in ER-induced apoptosis and the unfolded protein response (UPR). Real-time PCR and Western blot analysis demonstrated that the ER stress-induced proteins or genes GADD153 and GRP78 were upregulated by treatment with $P$. gingivalis 381 (Figures 2(a) and 2(b)), although there was not so drastic but still significant difference in GRP78 mRNA level. A significant increase in caspase-12 activity at $21 \mathrm{~h}$ after the addition of $P$. gingivalis 381 was also observed at MOI of $1: 10$ and $1: 10^{2}$ (Figure 2(c)). Furthermore, DNA fragmentation induced by $P$. gingivalis 381 infection was significantly suppressed by pretreatment with salubrinal and a caspase-12 inhibitor (Figure 2(d)). P. gingivalis 381 -induced caspase- 3 and caspase- 12 activities were also completely abrogated by pretreatment with salubrinal (Figure 2(e)). These data suggest that the ER stress response functions prior to $P$. gingivalis 381-mediated apoptosis.

3.3. P. gingivalis 381 Contributes to ER Stress-Induced Autophagy. Autophagy is essential for the removal of damaged organelles and long-lived cytosolic macromolecules to maintain energy homeostasis, and hence cell survival, under starvation conditions. When excessive, however, autophagy results in autophagic cell death. To examine the connection between $P$. gingivalis 381 infection and the stimulation of autophagy, we examined the expression of Atg6 (Beclin-1) and the colocalization of LC3-II (both are autophagosome markers). Real-time PCR and Western blot analysis demonstrated that LC3-II and Beclin-1 were upregulated by treatment with $P$. gingivalis 381 (Figures 3(a) and $3(b))$. In order to examine if autophagic signaling contributes to the protection against cell death, autophagy (a ratio of LC3-II/LC3-I) was suppressed by siRNA specific for LC3 (Figure 3(c)). P. gingivalis 381 significantly increased apoptosis in autophagy-deficient HUVEC. These results suggest that autophagy induced by $P$. gingivalis 381 protects HUVEC from apoptosis caused by the same bacteria.

Autophagy is also characterized by acidic vesicular organelle (AVO) formation, which can be assayed by acridine orange staining [17]. AVOs accumulated in the cytoplasm of HUVEC exposed to $P$. gingivalis 381 (Figure 3(d)); however, this was inhibited by the addition of salubrinal (Figure 3(d)). Furthermore, salubrinal suppressed $P$. gingivalis 381-induced LC3-II expression (Figure 3(e)). These data suggest that the ER stress response functions prior to autophagy induced by $P$. gingivalis 381 infection.

\section{Discussion}

In this study, we found that HUVEC challenge with high doses of $P$. gingivalis 381 for $21 \mathrm{~h}$ significantly exhibited suppression of viability. And it was found that this decrease in viability is cell death caused by endothelial apoptosis as evidenced by DNA fragmentation, annexin V staining, and caspase activity (Figures 1(a) and 1(d)). Furthermore, since the challenge with KDP136 did not induce DNA fragmentation, it was suggested that the apoptosis-inducing factor in P. gingivalis 381 may be caused by gingipain (Figure 1(b)). Caspase-3, caspase-8, and caspase- 9 were significantly activated by intermediate high doses of $P$. gingivalis 381 infection, suggesting that multiple signaling pathways were involved. Therefore, activation of these caspases may 


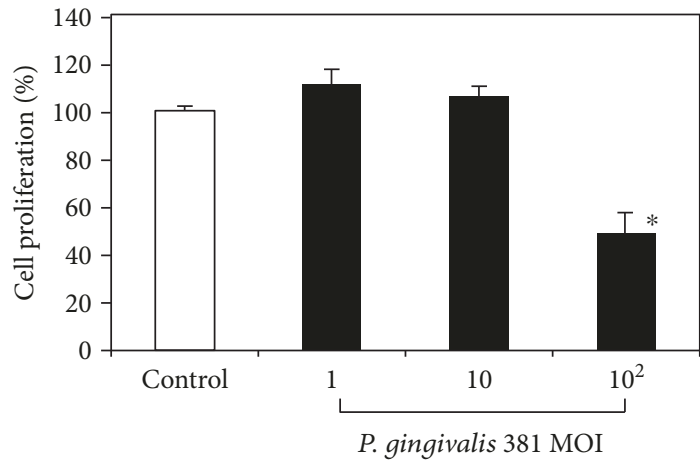

(a)

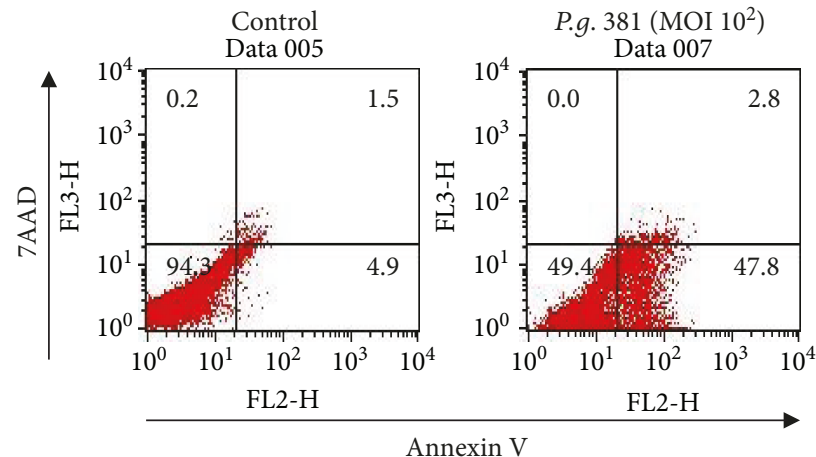

Annexin V

(c)

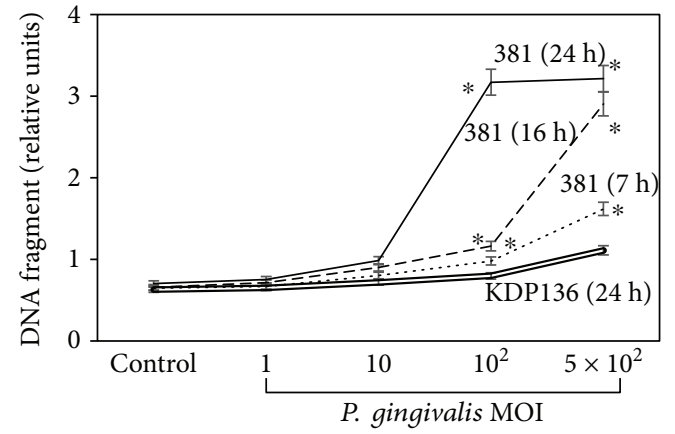

(b)

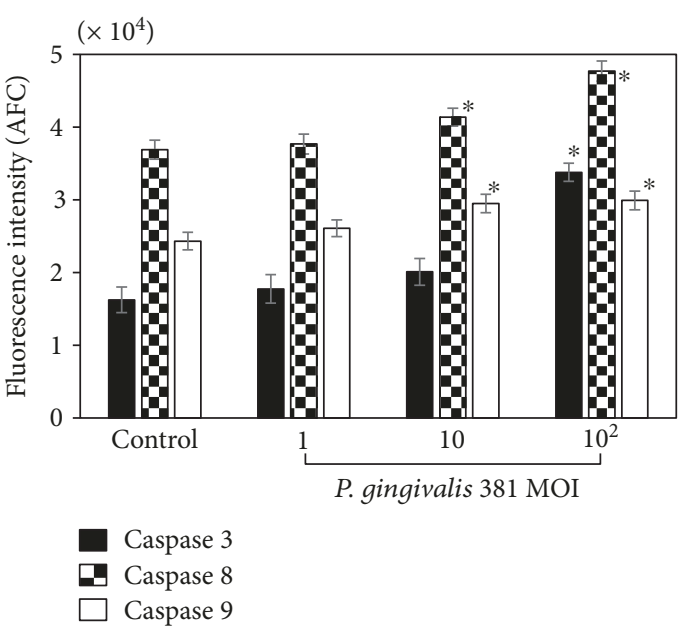

(d)

FIgURE 1: P. gingivalis infection inhibits cellular proliferation and induces apoptosis in HUVEC. (a) HUVEC were treated with $P$. gingivalis 381 at an MOI of $1: 1-1: 10^{2}$ for $21 \mathrm{~h}$. Cell viability was determined using a Cell Counting Kit- 8 (CCK-8). The data are expressed as the mean \pm SEM of 3 different experiments. ${ }^{*} p<0.05$ versus bacteria-free control cells. (b) HUVEC were treated with $P$. gingivalis 381 at an MOI of $1: 1-1: 5 \times 10^{2}$ for 7,16 , and $24 \mathrm{~h}$, and with KDP136 at an MOI of $1: 1-1: 5 \times 10^{2}$ for $24 \mathrm{~h}$. Cellular apoptosis was quantified by DNA fragmentation using the Cell Death Detection ELISA ${ }^{\text {PLUS }}$ Kit as described in Materials and Methods. Data are expressed as the mean \pm SEM of 3 different experiments. ${ }^{*} p<0.05$ versus bacteria-free control cells. (c) HUVEC were stained with annexin V-EnzoGold and 7-AAD after treatment with $P$. gingivalis 381 at an MOI of $1: 10^{2}$ for $21 \mathrm{~h}$, and then they were analyzed by flow cytometry. The figure is representative of three experiments with similar results. (d) HUVEC were treated with $P$. gingivalis 381 at an MOI of $1: 1-1: 10^{2}$ for $16 \mathrm{~h}$. Cell extracts were prepared, and caspase activities were measured. Data are expressed as the mean \pm SEM of 3 different experiments. ${ }^{*} p<0.05$ versus bacteria-free control cells.

contribute to endothelial dysfunction. Pyroptosis is a more recently recognized form of regulated cell death with morphological and biochemical properties distinct from necrosis and apoptosis [16]. However, the death of the HUVEC in this study was unrelated to pyroptosis since caspase-1 activity was not changed at any $P$. gingivalis 381 dose examined (data not shown).

In this study, $P$. gingivalis 381 induced the expression of a number of ER stress markers, including GADD153, GRP78, and caspase-12, indicating that $P$. ginigivalis 381 induces ER stress. The mRNA and protein levels of GADD153 and GRP78 were increased in MOI concentration dependently except for the mRNA level of GADD153, but the caspase12 activity was maximized at MOI (10) and decreased at MOI $\left(10^{2}\right)$. Because gingipain of $P$. gingivalis can cleave caspase-3 [18], cleavage of caspase-12 may be occurring at high concentration of MOI. Furthermore, since addition of either an ER stress inhibitor (salburinal) or an anti-caspase12 reagent significantly decreased $P$. gingivalis 381 -induced apoptosis in HUVEC, ER stress may occur upstream rather than downstream of apoptosis in cells exposed to high doses of $P$. gingivalis 381. Therefore, the activation of ER stress markers such as GADD153, GRP78, and caspase-12 will likely to happen at an early stage compared with the factors related to apoptosis. Induction of the mammalian UPR involves, in part, enhanced transcription of genes encoding ER chaperones, including BiP/GRP78, which serves to correct protein misfolding. The UPR, which serves to restore cellular homeostasis, induces the transcription of genes encoding antiapoptotic and proapoptotic proteins. Thus, severe or prolonged ER stress may induce apoptosis. Again, our data suggest that $P$. gingivalis 381 induces apoptosis after 

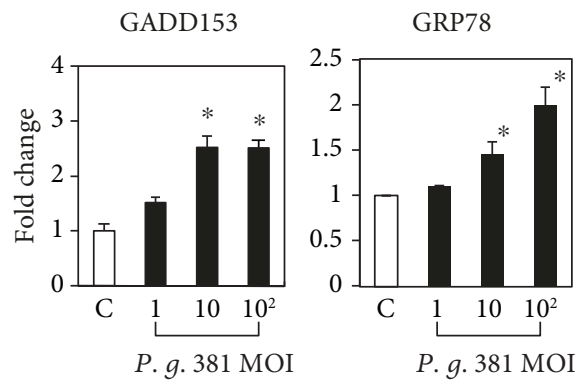

(a)

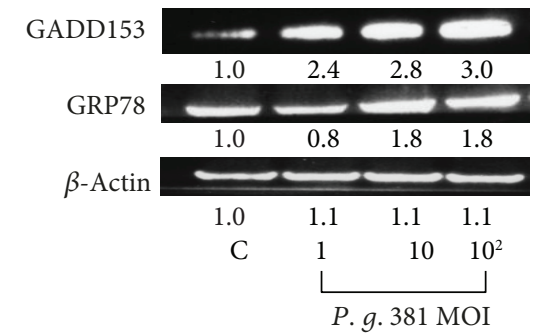

(b) $\left(\times 10^{3}\right)$

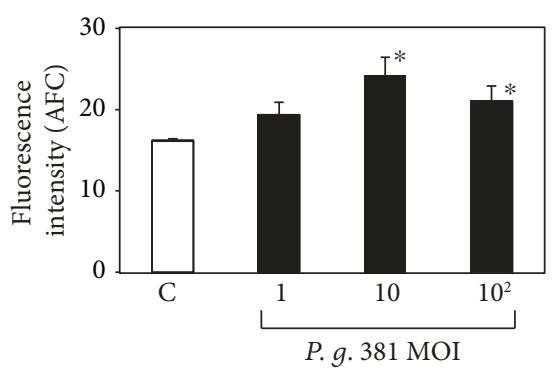

(c)

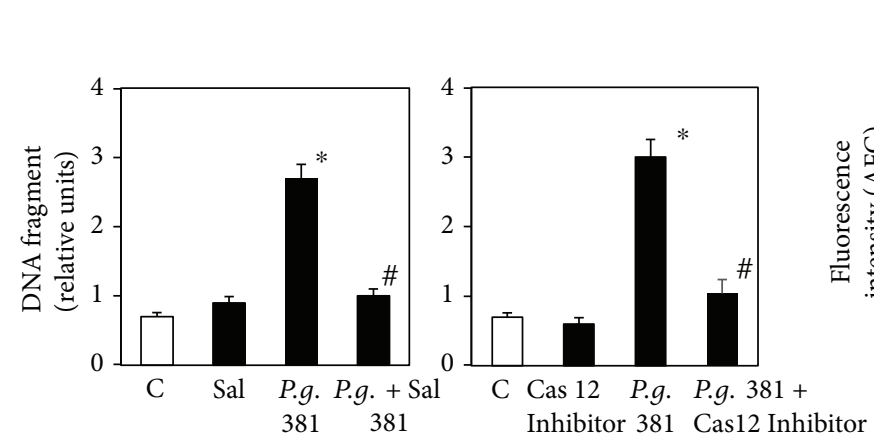

(d)

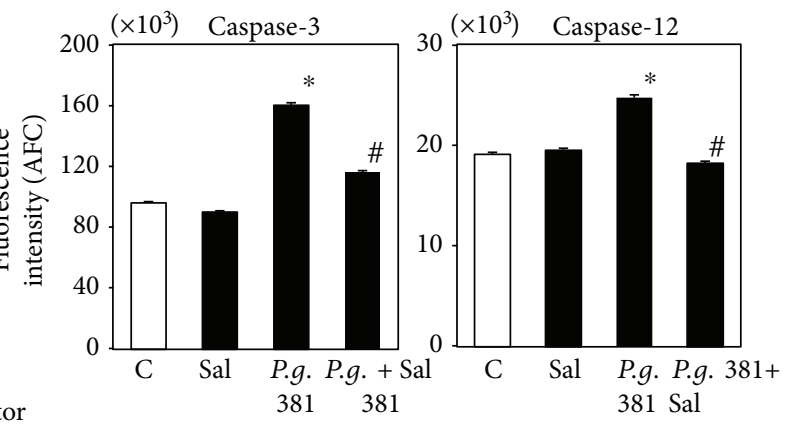

(e)

FIgURE 2: $P$. gingivalis 381 induces UPR-like response in HUVEC. (a) HUVEC were treated with P. gingivalis 381 at an MOI of $1: 1-1: 10^{2}$ for $8 \mathrm{~h}$. Quantitative real-time PCR analysis was performed for GADD153 and GRP78 relative to $\beta$-actin expression. Data are expressed as the mean \pm SEM of 3 different experiments. ${ }^{*} p<0.01$ versus bacteria-free control cells. (b) HUVEC were treated with $P$. gingivalis 381 at an MOI of $1: 1-1: 10^{2}$ for $21 \mathrm{~h}$. Whole cell lysates were subjected to SDS-PAGE, followed by Western blot analysis using antibodies to GADD153 and GRP78. Levels of $\beta$-actin were also detected as an internal control. The density of each band was measured by densitometry, and the relative band densities were calculated by comparing the band densities with those of the control. (c) HUVEC were treated with $P$. gingivalis 381 at an MOI of $1: 1-1: 10^{2}$ for $16 \mathrm{~h}$. Cell extracts were then prepared, and caspase-12 activity was measured. Data are expressed as the mean \pm SEM of 3 different experiments. ${ }^{*} p<0.01$ versus bacteria-free control cells. (d) HUVEC were pretreated with a caspase-12 or ER stress inhibitor for $1 \mathrm{~h}$ and then treated with P. gingivalis 381 at an MOI of $1: 10^{2}$ for $24 \mathrm{~h}$. The ratio of apoptotic cells to total cells was determined using the Cell Death Detection ELISA ${ }^{\text {PLUS }}$ Kit. Data are expressed as the mean \pm SEM of 3 different experiments. * $p<0.01$ versus bacteria-free control cells. ${ }^{\#} p<0.01$ versus bacteria-treated control cells. (e) HUVEC were pretreated with an ER stress inhibitor for $1 \mathrm{~h}$ and then treated with $P$. gingivalis 381 at an MOI of $1: 10^{2}$ for $16 \mathrm{~h}$. Cell extracts were then prepared, and caspase activity was measured. Data are expressed as the mean \pm SEM of 3 different experiments. ${ }^{*} p<0.01$ versus bacteria-free control cells. ${ }^{\#} p<0.01$ versus bacteria-treated control cells.

ER stress. ER stress has been demonstrated at all stages of atherosclerotic lesion development in ApoE knockout mice, and it is evident in human atherosclerotic lesions $[12,19,20]$. However, the relationship between P. gingivalis 381 infection and ER stress is yet to be determined. Our preliminary data indicated that the infection with a higher $P$. gingivalis 381 MOI induced reactive oxygen species (ROS) in HUVEC (data not shown). It is known that ROS induces the ER stress [21, 22]. Further, it was also found that lipopolysaccharide (LPS) could activate the ER stress [23]. Therefore, ROS and LPS release in HUVEC by infection with $P$. gingivalis might induce the ER stress to HUVEC. Indeed, the expression levels of UPRrelated genes were significantly higher in periodontitis compared with gingivitis lesions [24].

Accumulating evidence suggests that the ER plays an essential role not only in apoptosis but also in the regulation of autophagy [14, 25]. However, whether ER stress-mediated autophagy contributes to cell survival or cell death remains unclear. Here, we found that $P$. gingivalis 381-induced ER stress enhanced autophagy. The process of autophagosome formation depends on several autophagy proteins [26]. By translational modification of LC3, LC3-II (16 kDa) localizes exclusively to autophagosomal membranes and has been used as an autophagy marker [27]. We demonstrated that $P$. gingivalis 381 induced the expression of autophagy markers (e.g., Beclin-1, LC3-II, and AVOs), and that LC3-II and AVO expressions were inhibited by salubrinal. In addition, since specific suppression of LC3 by siRNA effectively downregulated LC3-II/LC3-I ratio and significantly increased the apoptosis in HUVEC upon stimulation with $P$. gingivalis 381, the autophagy may protect HUVEC from $P$. gingivalis 381-induced apoptosis. Most likely, autophagy under basal conditions plays an important role in cellular housekeeping, whereas induced autophagy may function as a death pathway, as suggested by our results. 

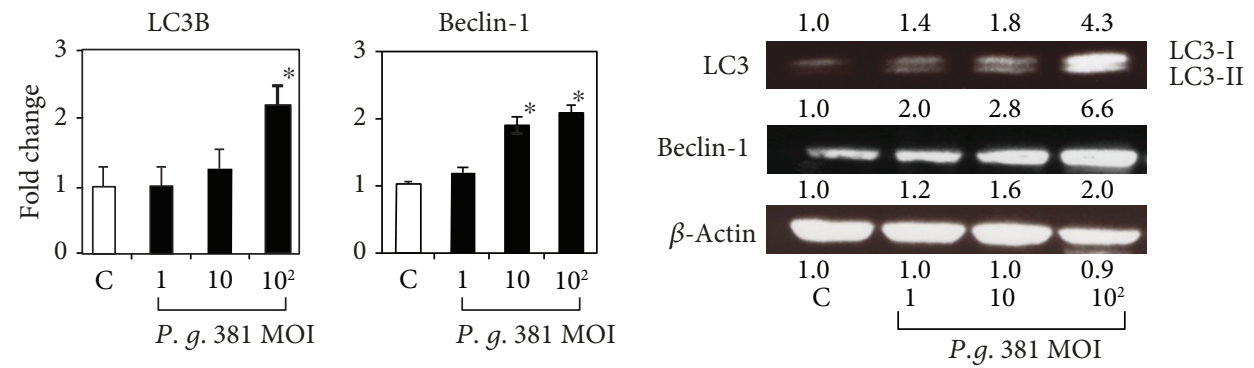

(a)

(b)
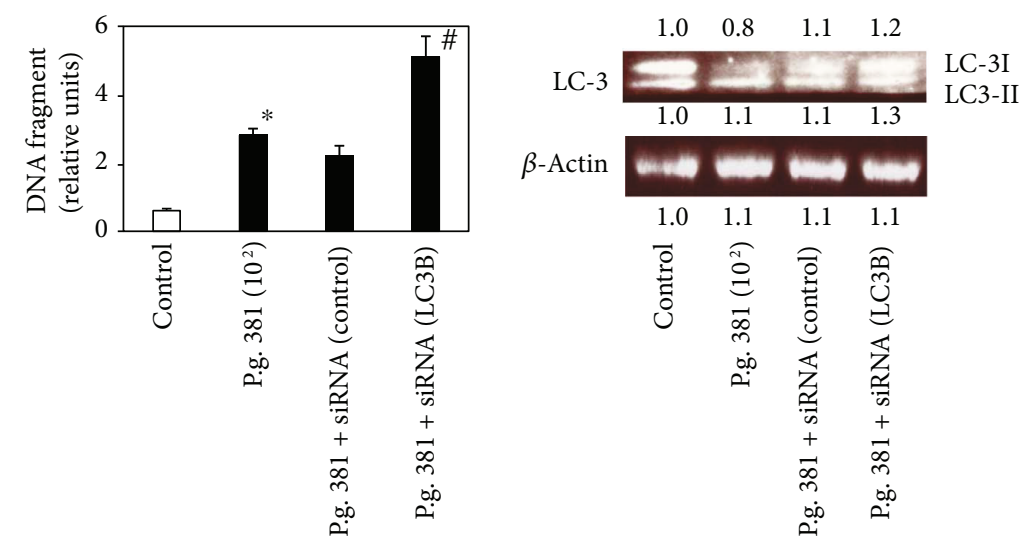

(c)

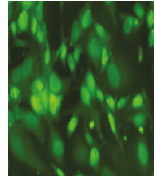

Control

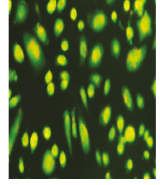

P. g. (MOI: $10^{2}$ )

381

(d)

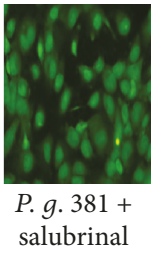

salubrinal

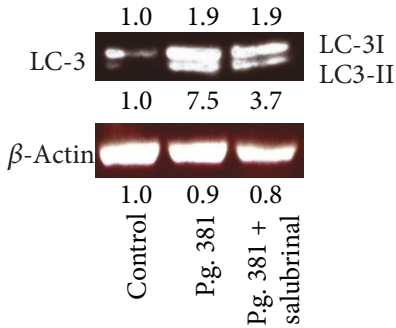

(e)

FIgURe 3: P. gingivalis 381 triggers ER stress-induced autophagy in HUVEC. (a) HUVEC were treated with P. gingivalis 381 at an MOI of $1: 1-1: 10^{2}$ for $8 \mathrm{~h}$. Quantitative real-time PCR analysis was performed for LC3B and Beclin-1 relative to $\beta$-actin expression. Data are expressed as the mean \pm SEM of 3 different experiments. ${ }^{*} p<0.01$ versus bacteria-free control cells. (b) HUVEC were treated with P. gingivalis 381 at an MOI of $1: 1-1: 10^{2}$ for $21 \mathrm{~h}$. Whole cell lysates were subjected to SDS-PAGE, followed by Western blot analysis using antibodies to LC3 and Beclin-1. Levels of $\beta$-actin were also detected as an internal control. The density of each band was measured by densitometry, and the relative band densities were calculated by comparing the band densities with those of the control. (c) HUVEC were transfected with $50 \mathrm{nM}$ of LC3B-specific siRNA or control siRNA and then stimulated with P. gingivalis 381 at an MOI of $1: 10^{2}$ for $8 \mathrm{~h}$ (real-time PCR) or $16 \mathrm{~h}$ (Western blotting). The ratio of apoptotic cells to total cells was determined using the Cell Death Detection ELISA ${ }^{\text {PLUS }}$ Kit. Data are expressed as the mean \pm SEM of 3 different experiments. ${ }^{*} p<0.01$ versus bacteria-free control cells. ${ }^{\#} p<0.01$ versus nontransfected or control siRNA-treated cells. The expression of LC3 was analyzed by Western blotting. Lysates were then immunoblotted with anti-LC-3. Levels of $\beta$-actin were also detected as an internal control. (d) HUVEC were pretreated with an ER stress inhibitor for $1 \mathrm{~h}$ and then treated with $P$. gingivalis 381 at an MOI of $1: 10^{2}$ for $8 \mathrm{~h}$. Acridine orange staining indicated that the ER stress inhibitor suppressed autophagic vacuolation induced by $P$. gingivalis 381. (e) HUVEC were pretreated with an ER stress inhibitor for $1 \mathrm{~h}$ and then treated with $P$. gingivalis 381 at an MOI of $1: 10^{2}$ for $21 \mathrm{~h}$. Whole cell lysates were subjected to SDS-PAGE, followed by Western blot analysis using antibodies to LC3. Levels of $\beta$-actin were also detected as an internal control. The density of each band was measured by densitometry, and the relative band densities were calculated by comparing the band densities with those of the control.

In summary, our data highlight the important relationships of ER stress and autophagy with apoptosis in HUVEC during $P$. gingivalis 381 exposure. Our data also provide putative mechanisms for $P$. gingivalis 381-induced endothelial dysfunction, as well as suggesting potential strategies for the prevention of $P$. gingivalis 381-induced endothelial impairment.

\section{Conclusions}

The experiments revealed that $P$. gingivalis 381 infection induces ER stress-mediated apoptosis followed by autophagic response that protects HUVEC from $P$. gingivalis 381mediated apoptosis, potentially amplifying proatherogenic mechanisms in the perturbed vasculature. 


\section{Data Availability}

The data used to support the findings of this study are available from the corresponding author upon request.

\section{Disclosure}

This work was presented at the following link: https://www. researchgate.net/publication/266772287_Porphyromonas_ gingivalis_induces_ER_stress-induced_apoptosis_and_auto phagy_in_HUVEC.

\section{Conflicts of Interest}

The authors report no conflict of interests.

\section{Acknowledgments}

The authors express their sincere thanks to M. Naito and K. Nakayama (Nagasaki University) for providing various mutant P. gingivalis strains. The authors thank Dr. Masafumi Yamamoto, Nihon University, for the great help and support. This study was supported by grants-in-aid for scientific research (22390398) from the Japan Society for the Promotion of Science, Strategic Research Base Development Program for Private Universities, 2010-2014 (S1001024), and an Academic Frontier Project for Private Universities matching fund subsidy (2007-2011) from the Ministry of Education, Culture, Sports, Science and Technology (MEXT), Japan.

\section{References}

[1] B. L. Pihlstrom, B. S. Michalowicz, and N. W. Johnson, "Periodontal diseases," Lancet, vol. 366, no. 9499, pp. 18091820, 2005.

[2] F. C. Gibson III, H. Yumoto, Y. Takahashi, H. H. Chou, and C. A. Genco, "Innate immune signaling and Porphyromonas gingivalis-accelerated atherosclerosis," Journal of Dental Research, vol. 85, no. 2, pp. 106-121, 2006.

[3] D. F. Kinane, M. P. Riggio, K. F. Walker, D. MacKenzie, and B. Shearer, "Bacteraemia following periodontal procedures," Journal of Clinical Periodontology, vol. 32, no. 7, pp. 708713, 2005.

[4] V. I. Haraszthy, J. J. Zambon, M. Trevisan, M. Zeid, and R. J. Genco, "Identification of periodontal pathogens in atheromatous plaques," Journal of Periodontology, vol. 71, no. 10, pp. 1554-1560, 2000.

[5] E. V. Kozarov, B. R. Dorn, C. E. Shelburne, W. A. Dunn, and A. Progulske-Fox, "Human atherosclerotic plaque contains viable invasive Actinobacillus actinomycetemcomitans and Porphyromonas gingivalis," Arteriosclerosis, Thrombosis, and Vascular Biology, vol. 25, no. 3, pp. e17-e18, 2005.

[6] B. R. Dorn, W. A. Dunn, and A. Progulske-Fox, "Porphyromonas gingivalis traffics to autophagosomes in human coronary artery endothelial cells," Infection and Immunity, vol. 69, no. 9, pp. 5698-5708, 2001.

[7] G. A. Roth, B. Moser, S. J. Huang et al., "Infection with a periodontal pathogen induces procoagulant effects in human aortic endothelial cells," Journal of Thrombosis and Haemostasis, vol. 4, no. 10, pp. 2256-2261, 2006.
[8] G. A. Roth, B. Moser, F. Roth-Walter et al., "Infection with a periodontal pathogen increases mononuclear cell adhesion to human aortic endothelial cells," Atherosclerosis, vol. 190, no. 2, pp. 271-281, 2007.

[9] Q. Xu, "The impact of progenitor cells in atherosclerosis," Nature Clinical Practice Cardiovascular Medicine, vol. 3, no. 2, pp. 94-101, 2006.

[10] A. Dardik, L. Chen, J. Frattini et al., "Differential effects of orbital and laminar shear stress on endothelial cells," Journal of Vascular Surgery, vol. 41, no. 5, pp. 869-880, 2005.

[11] E. Durand, A. Scoazec, A. Lafont et al., "In vivo induction of endothelial apoptosis leads to vessel thrombosis and endothelial denudation: a clue to the understanding of the mechanisms of thrombotic plaque erosion," Circulation, vol. 109, no. 21, pp. 2503-2506, 2004.

[12] J. Zhou, S. Lhotak, B. A. Hilditch, and R. C. Austin, "Activation of the unfolded protein response occurs at all stages of atherosclerotic lesion development in apolipoprotein E-deficient mice," Circulation, vol. 111, no. 14, pp. 1814-1821, 2005.

[13] N. Gurusamy and D. K. Das, "Is autophagy a double-edged sword for the heart?," Acta Physiologica Hungarica, vol. 96, no. 3, pp. 267-276, 2009.

[14] D. Senft and Z. A. Ronai, "UPR, autophagy, and mitochondria crosstalk underlies the ER stress response," Trends in Biochemical Sciences, vol. 40, no. 3, pp. 141-148, 2015.

[15] E. M. Creagh, H. Conroy, and S. J. Martin, "Caspase-activation pathways in apoptosis and immunity," Immunological Reviews, vol. 193, no. 1, pp. 10-21, 2003.

[16] T. Bergsbaken, S. L. Fink, and B. T. Cookson, "Pyroptosis: host cell death and inflammation," Nature Reviews Microbiology, vol. 7, no. 2, pp. 99-109, 2009.

[17] S. Paglin, T. Hollister, T. Delohery et al., "A novel response of cancer cells to radiation involves autophagy and formation of acidic vesicles," Cancer Research, vol. 61, no. 2, pp. 439-444, 2001.

[18] J. A. Kinane, M. R. Benakanakere, J. Zhao, K. B. Hosur, and D. F. Kinane, "Porphyromonas gingivalis influences actin degradation within epithelial cells during invasion and apoptosis," Cellular Microbiology, vol. 14, no. 7, pp. 1085-1096, 2012.

[19] P. S. Gargalovic, N. M. Gharavi, M. J. Clark et al., "The unfolded protein response is an important regulator of inflammatory genes in endothelial cells," Arteriosclerosis, Thrombosis, and Vascular Biology, vol. 26, no. 11, pp. 2490-2496, 2006.

[20] J. Gao, Y. Ishigaki, T. Yamada et al., "Involvement of endoplasmic stress protein C/EBP homologous protein in arteriosclerosis acceleration with augmented biological stress responses," Circulation, vol. 124, no. 7, pp. 830-839, 2011.

[21] M. Yokouchi, N. Hiramatsu, K. Hayakawa et al., "Involvement of selective reactive oxygen species upstream of proapoptotic branches of unfolded protein response," The Journal of Biological Chemistry, vol. 283, no. 7, pp. 4252-4260, 2008.

[22] Y. Tagawa, N. Hiramatsu, A. Kasai et al., "Induction of apoptosis by cigarette smoke via ROS-dependent endoplasmic reticulum stress and CCAAT/enhancer-binding proteinhomologous protein (CHOP)," Free Radical Biology \& Medicine, vol. 45 , no. 1 , pp. 50-59, 2008.

[23] S. Alhusaini, K. McGee, B. Schisano et al., "Lipopolysaccharide, high glucose and saturated fatty acids induce endoplasmic reticulum stress in cultured primary human adipocytes: salicylate alleviates this stress," Biochemical and Biophysical Research Communications, vol. 397, no. 3, pp. 472-478, 2010. 
[24] H. Domon, N. Takahashi, T. Honda et al., "Up-regulation of the endoplasmic reticulum stress-response in periodontal disease," Clinica Chimica Acta, vol. 401, no. 1-2, pp. 134-140, 2009.

[25] M. Ogata, S. Hino, A. Saito et al., "Autophagy is activated for cell survival after endoplasmic reticulum stress," Molecular and Cellular Biology, vol. 26, no. 24, pp. 9220-9231, 2006.

[26] T. Shintani and D. J. Klionsky, "Autophagy in health and disease: a double-edged sword," Science, vol. 306, no. 5698, pp. 990-995, 2004.

[27] Y. Kabeya, N. Mizushima, T. Ueno et al., "LC3, a mammalian homologue of yeast Apg8p, is localized in autophagosome membranes after processing," The EMBO Journal, vol. 19, no. 21 , pp. $5720-5728,2000$. 


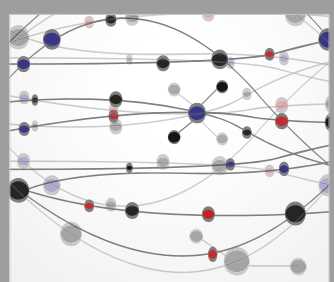

The Scientific World Journal
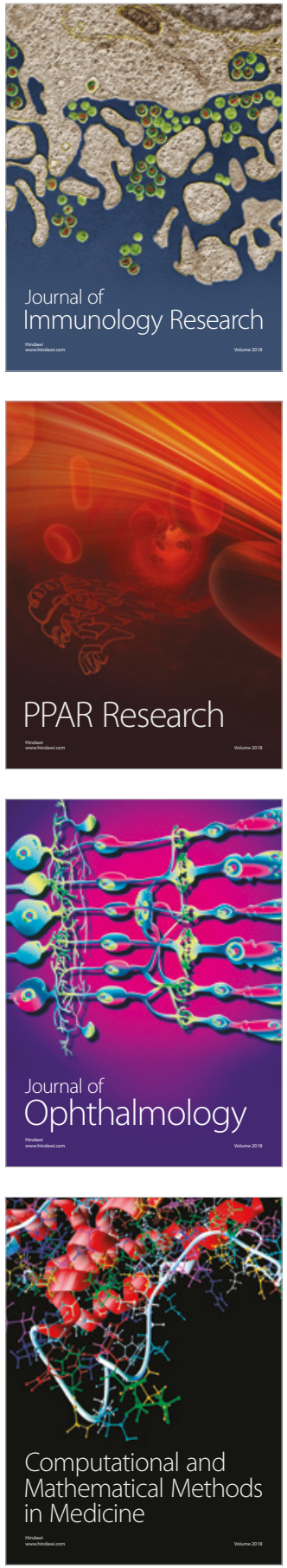

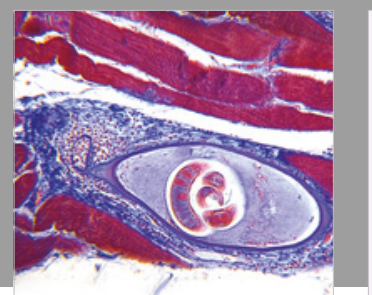

Gastroenterology Research and Practice

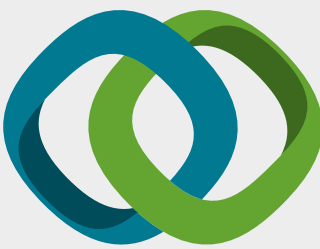

\section{Hindawi}

Submit your manuscripts at

www.hindawi.com
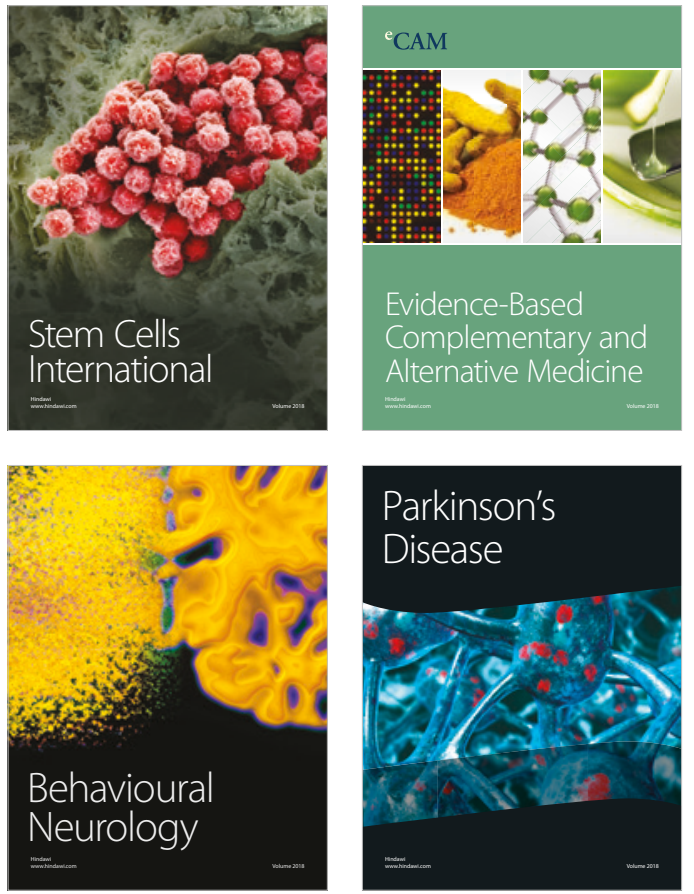

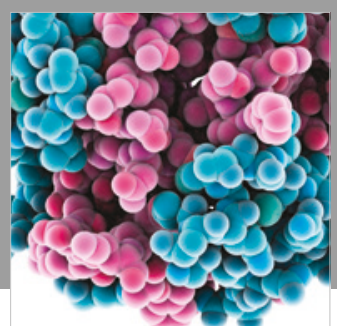

ournal of

Diabetes Research

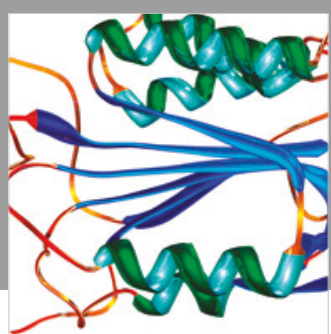

Disease Markers
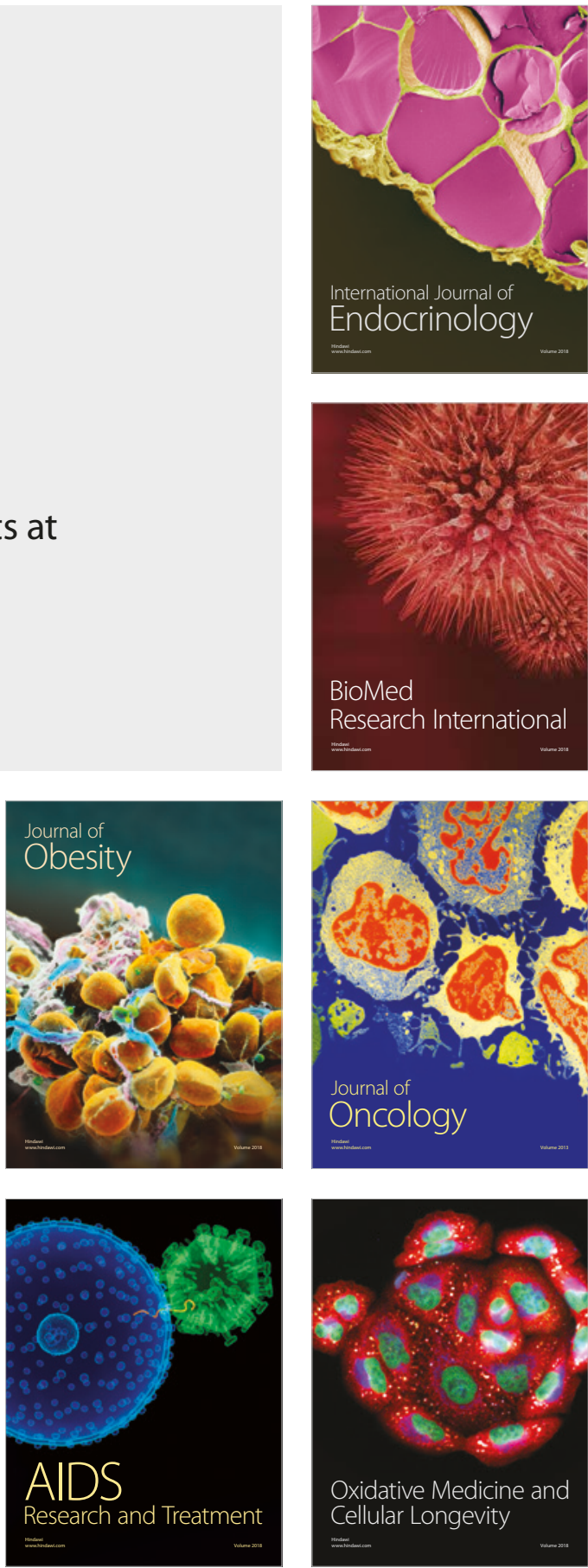